\title{
PREVALENCIA DEL DESEO SEXUAL INHIBIDO EN MUJERES EN EDAD FÉRTIL Y FACTORES RELACIONADOS
}

\author{
PREVALENCE OF INHIBITED SEXUAL DRIVE IN WOMEN ACTIVELY \\ REPRODUCTIVE AND RELATED FACTORS
}

\author{
MÓNICA CHARNAY REBOLLEDO* y ELENA HENRIQUEZ FIERRO**
}

\begin{abstract}
RESUMEN
Estudio descriptivo correlacional cuyo objetivo fue conocer la magnitud del deseo sexual inhibido y factores que lo influyen, en mujeres en edad fértil usuarias del Sistema Municipalizado de Salud de la comuna de Concepción. La muestra estudiada correspondió a 367 mujeres. Se aplicó un cuestionario elaborado por la autora que mide las variables biosociodemográficas y factores que influyen en la presencia de deseo sexual inhibido y un instrumento de Master, Johnson y Kolodny, "Autovaloración del Deseo Sexual Inhibido", modificado y adaptado por la autora. Los resultados nos indican que un 34,9\% de las mujeres presenta deseo sexual inhibido, que existe relación estadísticamente significativa entre la variable, nivel educacional de la mujer y de la pareja, situación laboral de la pareja, satisfacción de las necesidades básicas, satisfacción sexual, satisfacción con la relación de pareja y la variable dependiente deseo sexual inhibido. Para la asociación estadística, se utilizó chi cuadrado de Pearson.
\end{abstract}

Palabras claves: Prevalencia del deseo sexual inhibido, mujer y factores relacionados.

\begin{abstract}
The objective of this descriptive correlational study was to know the magnitude of the inhibited sexual desire and the factors that affect it, in women reproductively active, registered at the Municipal Health System in Concepción city. The population studied comprised 367 women. A questionnaire made by the author measuring biosocialdemographic variables and factors affecting the inhibited sexual drive and a modified Master, Johnson and Kolodny's instrument on "Self evaluation of Inhibited Sexual Drive" were applied. The results indicate that $34.9 \%$ of women present Inhibited Sexual Drive and that there is a statistically significant relation between the variables, women's schooling, couple's schooling, couple's laboral situation, basic needs satisfaction, sexual satisfaction, satisfaction about the couple relationship and the inhibited sexual drive dependent variable. For the statistical association Pearson Chi square was used.
\end{abstract}

Keywords: Prevalence, inhibited sexual desire, woman reproductively active, related factors.

Recepcionado: 29. 04.2003. Aceptado: 23.06.2003.

\section{INTRODUCCIÓN}

El ser humano es una unidad integral, compuesta por varias dimensiones; una de estas dimensiones es la sexualidad, construcción social que se origina en el propio individuo, proceso que interpreta y adscribe un significado cultural a los pensamientos, a las conductas y condiciones sexuales, y en donde la experiencia que cada persona pueda tener de la sexualidad está mediada por factores biológicos, psicológicos, socioeconómicos, culturales, éticos y religiosos o espirituales, etc. (Zeidenstein, 1999).

Esto ha originado que sea una de las dimensiones del ser humano que históricamente ha creado conflicto para su estudio, ya que existen diversas opiniones y enfoques respec-

*Profesora Asistente, Magister en Enfermería Mención Salud Comunitaria, Departamento de Enfermería, Facultad de Medicina Universidad de Concepción, Chile, E-mail: mocharna@udec.cl

**Profesora Titular, Magister en Enfermería Mención Salud Comunitaria, Departamento de Enfermería, Facultad de Medicina Universidad de Concepción, Chile, E-mail: ehenriqu@udec.cl 
to a su significado, a su importancia y a las relaciones con los otros aspectos de la naturaleza humana. De esto es que cada cultura aborda en forma distinta esta expresión natural (Pino, 1992).

A través de su historia, el ser humano primitivamente vivía en un plano meramente biológico, agobiado por la lucha diaria por la supervivencia, y la mujer, protectora innata, buscaba la seguridad de su prole. La sexualidad era en ese entonces la necesidad imperante de saciar un impulso biológico que los llevaba a la cópula, con los objetivos principales: la reproducción y el mitigar conflictos de la especie, dada la conocida agresividad del macho (Morris, 2000).

Durante siglos la mujer fue menospreciada, postergada para cumplir el rol de hija obediente, que acataba la decisión de su padre acerca de con quién vivir su sexualidad y maternidad, esposa sumisa y madre abnegada, reprimiendo la expresión de su sexualidad por vergüenza y temor, ya que actuaba en función de lo que el hombre requería (Worth, 1999).

Sólo a partir de la mitad del siglo XX, coincidiendo con la aparición de la píldora anticonceptiva, se marca el inicio de una nueva era de la sexualidad femenina, donde se sentó la base para situar a la mujer en un plano de igualdad con el hombre (Zeidenstein, 1999).

La angustia de la maternidad no deseada, en el presente al igual que en el pasado, sigue siendo una causal importante de aparición de disfunciones sexuales, en especial de una falta de deseo sexual; por consiguiente, al disminuir esta angustia aumenta la posibilidad de disfrutar del sexo, ya que la mujer se permite sentir placer durante las relaciones sexuales (Jiménez, 1999).

Pero esta revolución tuvo resultados ambivalentes, a pesar de que se estimuló a la sociedad a ser menos puritana y estricta con el sexo, también obligó de cierta forma a la mujer a la disponibilidad permanente para los hombres, ya que aún en nuestros tiempos persiste la idea que las mujeres no tienen las mismas necesidades y deseos sexuales que los hombres, donde se reconoce que el hombre goza de una satisfacción del sexo inherente a su sexo biológico (Dixon, 1999).

En consecuencia, la sexualidad en general y la femenina en particular está mediatizada por la cultura, la época, los valores presentes en las distintas sociedades. Es así como existen sociedades permisivas que le han dado a la mujer la oportunidad de cultivar una sexualidad placentera, igualitaria a la del varón, en contrapartida con aquellas culturas represivas donde se ha convertido a la mujer en un ser asexuado, reprimido, donde no se les alienta ni apoya para que tomen decisiones relativas a su sexualidad, elección de su pareja, planificación familiar y, menos aún, que se empoderen de su deseo sexual (Worth, 1999).

Particularmente en Chile, si sumamos a lo anterior la diferencia genérica condicionada durante siglos por culturas predominantemente machistas como la nuestra, tendremos un grupo importante de personas, mujeres y jóvenes, con acceso restringido a información acerca de cómo vivir plenamente su sexualidad ( Rodó, 1992).

La OMS define la salud sexual como "la experiencia del proceso continuo de bienestar físico, psicológico y sociocultural relacionado con la sexualidad", y para que la salud sexual se alcance es necesario que los derechos sexuales de las personas -como el derecho al placer sexual, a la expresión sexual emocional, a información basada en el conocimiento científico, a la educación sexual integral, a la atención de la salud sexual- se reconozcan y se garanticen (OMS, 2000).

Enfermería, como ciencia y profesión, se interesa por todo lo que le acontece al individuo y a su entorno, y cómo éste es influenciado por los aspectos sociales, políticos, culturales, genéricos, familiares y ambientales.

Lo anterior ha motivado a realizar una investigación en torno a la consulta que más se repite en la literatura especializada chilena y extranjera, esto es, la disminución del deseo sexual, para conocer la realidad de las mujeres chilenas usuarias del Sistema Municipalizado de Salud de la comuna de Concepción, ya que sólo se poseen estadísticas extranjeras. A fin de contribuir con los resul- 
tados al quehacer del profesional de enfermería en esta área para planificar estrategias de solución, ya sea a través de la orientación, la educación de la mujer y su pareja o la referencia a otro profesional para la terapia correspondiente cuando el caso así lo amerite.

\section{OBJETIVO GENERAL}

Conocer la magnitud del Deseo Sexual Inhibido y los factores que la influyen, en mujeres en edad fértil, usuarias del Sistema Municipalizado de Salud de la comuna de Concepción, que están en control en los policlínicos de planificación familiar.

\section{MARCO REFERENCIAL}

Para lograr sobrevivir y desarrollarse en forma adecuada, el ser humano necesita dar satisfacción a ciertas necesidades, que se ordenan en forma jerárquica, donde las necesidades fisiológicas son más imperiosas que la de seguridad. Éstas, a su vez, son más apremiantes que la necesidad de amor y de ser aceptado por un grupo organizado, las cuales son más perentorias que la necesidad de autoestima y de estimación por otros. En este orden piramidal la necesidad de autoactualización ocupa el último lugar. Las necesidades fisiológicas son, por lo tanto, primordiales, deben ser satisfechas antes que otras, ya que su privación produce una carencia desesperada que domina el organismo. Cada necesidad fisiológica está ordenada además en una subjerarquía que también cumple con ese postulado, por ejemplo, en el caso de las necesidades fisiológicas partiendo con la oxigenación y finalizando con la necesidad sexual (Maslow, 1963, 1985). Además, si se considera que la sexualidad es un proceso evolutivo e influenciado por numerosos estímulos, se debe decir también que requiere de un aprendizaje que será más o menos positivo, dependiendo del apoyo que se le brinde durante su trayectoria de maduración y sociabilización (Barra, 1999).
Socialmente, la sexualidad femenina se percibe desde una perspectiva del cuerpo y su utilidad, basándose en las modificaciones de éste y considerándolo como un cuerpo para otros, para la procreación o para el goce del hombre; reafirmando esta concepción de sexualidad para otros, donde la cultura exacerba el aspecto sexual en la vida de una mujer en perjuicio de otras cualidades por desarrollar, impide igualmente que esta sexualidad sea verdaderamente suya (Rivera, 1993).

Habitualmente se considera que la actividad sexual es algo natural, sin embargo un porcentaje alto de individuos, en algún momento de su vida, relata presentar alguna alteración; estos trastornos impiden disfrutar satisfactoriamente de la actividad sexual. Se denominan disfunciones sexuales y se define la disfunción como "toda alteración persistente de los patrones normales de interés y de respuesta sexual"' (Hawton, 1988).

El deseo sexual corresponde a una tendencia o motivación, a exponerse y/o a buscar activamente circunstancias que aumentan la probabilidad de llevar a cabo conductas autoéroticas o intercambios sexuales y sentirse receptivos a éstos (Martínez, 1999). Y se define deseo sexual hipoactivo (antes denominado deseo sexual inhibido) como inhibición persistente y profunda del deseo sexual (Flores, 2000).

Las personas hipoactivas no son atraídas por la posibilidad de tener contacto sexual alguno, su impulso sexual es bajo, muchas de ellas no tienen fantasías de contenido erótico o sexual (Labrador, 1994). La existencia de alteraciones en aquellos factores que activan la respuesta sexual, como por ejemplo la experiencia pasada en sexualidad, repercute en la disminución de la disposición o deseo de concretar una conducta sexual como es el coito (Sarquis, 1993). La conducta sexual y la sexualidad pueden ser estudiadas desde distintas perspectivas o enfoques, cada uno de los cuales aporta aspectos diferentes y a su vez complementarios (Rosenzvaig, 1994).

Para esta investigación, se utilizó como marco referencial teórico el modelo de la conducta sexual trabajado por J.A. Carrobles, lla- 
mado Modelo Secuencial de la Conducta Sexual Humana. Este modelo parte de la premisa de que la conducta sexual humana es un fenómeno complejo, que está determinado por múltiples determinantes biopsicosociales, las cuales se pueden agrupar en: biológicas, psicológicas y socioculturales. Los hechos pueden ser de origen innato o adquirido y pueden tener una situación temporal variable, es decir, remotos, presentes o inmediatos. La conducta sexual es la resultante de los factores antes mencionados y de las respuestas mediadoras del sujeto, y tiene consecuencias sobre el individuo (Carrobles, 1990).

En el presente estudio se ha contemplado una serie de variables independientes que se integran en los factores biológicos, psicológicos y socioculturales; estos dos últimos se han fusionado con el objeto de facilitar la integración de las variables en estudio al modelo original, ya que en algunos casos las variables se superponen. En los factores biológicos se incluyeron: la edad de la mujer, edad de su pareja, el método anticonceptivo utilizado y la edad del menor de los hijos. En los factores socioculturales se incluyeron: años de convivencia, nivel educacional de mujer y su pareja, ocupación de la mujer, condición laboral de la pareja, religión, fuente de información sexual. El instrumento utilizado en este estudio, "autovaloración del deseo sexual inhibido" de Masters, Johnson y Kolodny (1996), considera los siguientes factores biosicosociales: disposición positiva para las relaciones sexuales, iniciativa de la mujer, autoestima, percepción de pasión por parte de la mujer, diferencias de impulso sexual, fantasías sexuales, desacuerdos en la frecuencia de las relaciones.

\section{REFERENTE EMPIRICO}

Durante los últimos años han acontecido cambios en la posición de las mujeres en la sociedad, lo que ha determinado un aumento progresivo de la producción científica relacionada con los distintos aspectos de la sexualidad y de género, destacándose la lí- nea de género y estudios de la mujer (Palacios, 1995).

Laumann y col. (1999) investigaron las disfunciones sexuales en una muestra de la población general de EE.UU. Este estudio reveló que el grupo de mujeres y hombres solteros reporta una y media veces más problemas sexuales que aquellos casados, el $42 \%$ de las mujeres que no terminó los estudios divulgó carecer de deseo sexual, y sólo el $24 \%$ de las mujeres que terminó sus estudios reportó deseo sexual hipoactivo. Aquellas mujeres de ingresos económicos más bajos manifestaron experimentar con mayor frecuencia deseo sexual bajo. En aquellas familias donde existían niños que alteraban de alguna forma el grado de intimidad de la pareja, reportaron índices más altos de disfunciones sexuales. Fortín (1994) estudió en 1992, en Canadá, el rol de la intimidad y la autopercepción sexual en mujeres y hombres que viven como parejas, concluyendo que la satisfacción sexual está relacionada principalmente a la percepción de la satisfacción marital por parte de la mujer y de su pareja, y la intimidad en las relaciones. McCabe y col. (1997) realizaron un estudio para evaluar la asociación entre intimidad, calidad de vida y disfunción sexual, en Australia; encontraron que los individuos disfuncionales presentaron menor grado de intimidad y menor calidad de vida que los individuos funcionales. Sánchez-Bravo y col. (1997), en México, determinaron cómo se afectan los niveles de autoconcepto y satisfacción marital, encontraron que las disfunciones sexuales están estrechamente relacionadas con un bajo autoconcepto, así como la disminución en la satisfacción marital. Díaz y col. (1999) desarrollaron una investigación en Brasil. De un total de 378 mujeres, el 15\% reportó nunca tener deseo sexual y un $85 \%$ reportó tener deseo sexual frecuentemente o a veces. La calidad de la relación sexual en estas mujeres fue considerada en un $9,8 \%$ como mala, el $50 \%$ de las mujeres que tenían problemas sexuales los atribuían al uso de métodos anticonceptivos. Goldmeier, Judd \& Schroeder (2000) estudiaron la prevalencia de disfun- 
ciones sexuales en Inglaterra; el estudio arrojó alta frecuencia de disfunción sexual (20\%). Abrantes, Freitas y Dimenstein (2001) realizaron una investigación cualitativa en el distrito Sanitario Norte del Municipio de Natal, Brasil; los discursos tradujeron una vida sexual marcada por una falta de deseo, el 54\% de las mujeres manifiestan "no tener deseos de tener relaciones sexuales". En 1987 Andrea Rodó realizó una investigación en mujeres pobladoras de Santiago, las conclusiones de este trabajo indican que el placer corporal se asocia mayoritariamente al descanso físico, la asociación de la sexualidad al placer erótico y a la sensualidad es minoritaria, dos tercios de las entrevistadas asumen positivamente el ser una fuente de placer para su pareja, aunque para ellas la relación sexual no sea satisfactoria. Aliaga, P. y col. (2000) estudiaron 182 mujeres, atendidas en la unidad de Sexualidad Humana del Hospital Clínico de la Universidad de Chile. El 72\% consulta por disfunciones sexuales generales, de las cuales un 38\% reporta deseo sexual inhibido. En el año 2002, en Chile, la Comisión Nacional del SIDA del Ministerio de Salud pone a disposición de la comunidad los primeros análisis del "Estudio Nacional de Comportamiento Sexual". Del total de 3.151 mujeres, el 70,7 \% informa que ha conversado temas íntimos al menos en los últimos 12 meses, los interlocutores tienden a ubicarse casi exclusivamente entre la pareja (56\%), los amigos $(23 \%)$ y los parientes $(14,9 \%)$. Los profesionales de la salud juegan un rol muy débil como interlocutores $(1,0 \%)$, los casados con hijos menores conversarían con su pareja y sus amigos, los casados con hijos mayores conversarían con su pareja e hijos. En términos de estrato socioeconómico, el estrato bajo sólo posee la menor experiencia de conversaciones sobre intimidad.

\section{MATERIAL Y METODOS}

Investigación descriptiva, correlacional.

\section{Población}

Unidad de análisis. Mujer en control de planificación familiar en los consultorios municipalizados de la comuna de Concepción que cumple con los criterios de exclusión establecido.

Muestra. El muestreo fue de tipo prospectivo estratificada por centro de salud, según peso porcentual por cada uno de los centros; contempló a 367 mujeres en control en los policlínicos de planificación familiar del sistema municipalizado de la comuna de Concepción y para su obtención se consideraron los siguientes criterios de exclusión.

Criterios de exclusión. Mujeres con patologías crónicas, como hipertensión, diabetes. Mujeres con parejas que presentan alguna patología aguda agregada. Mujeres con pareja que presentan alguna patología crónica agregada como hipertensión, alcoholismo, diabetes. Mujeres con convivencia inestable de menos de tres años. Mujeres menores de 21 años y mayores de 45 años. Mujeres que no consintieron en participar en el diseño.

\section{Instrumento recolector de datos}

Cuestionario:

Parte A. Elaborado por la autora y que mide las variables biosociodemográficas y algunos factores que podrían estar influyendo en la presencia de deseo sexual inhibido.

Parte B. Cuestionario elaborado y probado por Master, Johnson y Kolodny, publicado en el libro Eros los mundos de la sexualidad en 1996, denominado "Autovaloración del deseo sexual inhibido", instrumento compuesto por 15 afirmaciones tipo diferencial semántico con puntuación de 1 a 9 pts. en cada ítem.

Procesamiento de los datos. El instrumento recolector de los datos fue codificado por la autora, utilizando el programa computacional Excel 97, luego los datos fueron procesa- 
dos con el programa estadístico computacional SPSS 10.1.

Validez y confiabilidad. El instrumento parte A fue validado por expertos en el área de la sexualidad en Chile. El instrumento parte B, "Autovaloración del deseo sexual Inhibido" está validado por sus autores (Master, W.; Johnson, V.; Kolodny, R., 1996).

Para medir la confiabilidad interna de los instrumentos se realizó una prueba piloto, en ella se evidenció poca comprensión de 6 afirmaciones del instrumento "Autovaloración del deseo inhibido" $(1,2,3,7,9,12)$, por estar enunciadas en carácter negativo, por lo que se modificó dándole un carácter positivo. La puntuación final no sufre variación ya que la conversión del puntaje es automática con el programa SPSS 10. La confiabilidad correspondió a un alpha de Cronbach de 0.7748 .

\section{RESULTADOS}

La distribución frecuencial y porcentual del deseo sexual inhibido obtenido en la escala de "Autovaloración de deseo sexual inhibido" nos muestra que del total de las mujeres, el $65,1 \%$ se autovalora con deseo sexual sin inhibición, el $28,1 \%$ se autovalora con un deseo sexual medianamente inhibido y el 6,8 \% presentan deseo sexual altamente inhibido.

Gráfico 1. Distribución frecuencial y porcentual del deseo sexual inhibido obtenido en la escala de "Autovaloración del deseo sexual inhibido".

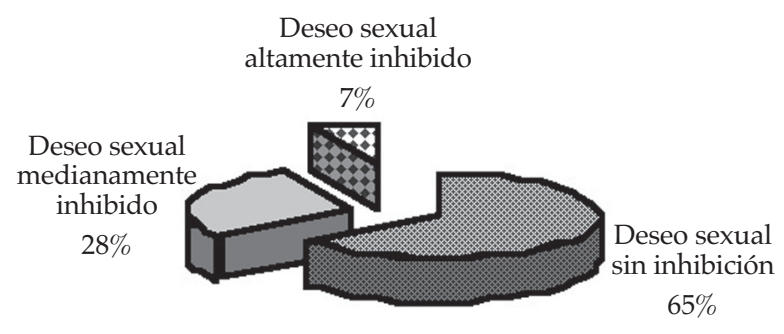

Fuente: Prevalencia deseo sexual inhibido y factores relacionados.

El perfil de la muestra corresponde a $3 / 4$ partes de las mujeres de 20 a 35 años y la pareja en similar proporción, la mitad de la muestra tiene entre 3 y 9 años de convivencia. Las tres cuartas partes tiene hijos menores de 6 años. Más de $1 / 4$ de las mujeres estudiadas no tiene espacio para la intimidad de pareja. La mitad de las mujeres usa anticonceptivos orales, una tercera parte utiliza dispositivo intrauterino, el resto utiliza preservativo, fue esterilizada o no usa nada. El $10 \%$ no terminó la escolaridad básica, el 51\% completó enseñanza media, el 19\% no completó la enseñanza media y el 19\% tiene estudios superiores; la escolaridad de las parejas de las mujeres se distribuyó de igual forma. El 69,8\% de las mujeres es dueña de casa, el $26,7 \%$ tiene trabajo remunerado y el
3,5\% es estudiante. En cuanto a la situación laboral de las parejas, el $84,7 \%$ tiene trabajo remunerado y el 15,3\% es cesante. El 67,6\% de las mujeres según su propia percepción cubre sus necesidades básicas esenciales, el $32,4 \%$ no da satisfacción a sus necesidades básicas. En relación a la fuente de información sexual, el 16,1\% dice haber aprendido de sexualidad de ambos padres, el 13,1\% la recibió sólo de la madre, el 0,3\% sólo del padre, el 25,6\% de las amigas, el 40,3\% de su pareja, el 48,5\% recibió educación de sus profesores, el 20,7\% de sus compañeros de estudios, el 53,7\% aprendió sexualidad de profesionales del área de la salud, el 86,6\% dice haber aprendido con los medios de comunicación. El 29,2\% de las mujeres profesa la religión evangélica, el 59,4\% profesa la religión 
católica, el 3,5\% otra religión y el 7,9\% no profesa ninguna. En relación a la percepción que tienen las mujeres de la satisfacción sexual, el 7,1\% manifiesta estar altamente insatisfechas, el 49,6\% manifiesta estar medianamente satisfecha y el $46,3 \%$ se considera altamente satisfecha. En cuanto a la percepción de la satisfacción con la relación de pareja sin considerar la vida sexual, el 5,4\% se percibe con insatisfacción, el 48,2\% la perciben como medianamente satisfechas y el $46,3 \%$ perciben como altamente satisfactoria su vida de pareja sin considerar su vida sexual.

\section{DISCUSIÓN}

Los resultados de este estudio muestran que el 34,9\% de las mujeres encuestadas presenta, en algún grado, inhibición del deseo sexual; estas cifras coinciden con los hallazgos obtenidos en investigaciones extranjeras. Labrador en 1987 obtuvo como resultado en su investigación sobre la prevalencia de disfunciones sexuales, en una muestra de la población de España, un 35\% de falta de interés por el sexo. En forma similar, Flores C. (2000), en su revisión de dos décadas de estadísticas de disfunciones sexuales, reportó como promedio un $38 \%$ de disminución del deseo sexual. Es importante destacar que estas mujeres no consultaron por alguna problemática sexual. En Chile, en un estudio realizado en el Hospital Clínico de la Universidad de Chile por Aliaga P. y col. (2000), encontraron un $38 \%$ de prevalencia de deseo sexual hipoactivo en mujeres consultantes en la unidad de sexualidad humana de la citada institución.

En relación a las variables edad de la mujer y edad de su pareja, el estudio indicó que, a mayor edad, se presenta en mayor porcentaje deseo sexual inhibido; esta frecuencia aumenta cuando las parejas tienen entre 40 y 45 años; se observa asimismo que, después de 10 años de convivencia estable, las mujeres reportan deseo sexual inhibido en mayor proporción que aquellas con menos años de vida en común, pero, a pesar del alto porcentaje $(40 \%)$, la asociación no es estadísticamente significativa. Abrantes y col. (2001), en su estudio de las necesidades de salud de las mujeres en las fabelas en Brasil, concluyeron que el 54\% de las mujeres encuestadas utilizaban el sexo como una estrategia para resguardar el vínculo de pareja; este hallazgo se repite en el estudio de Rodó (1987), donde las mujeres otorgan su sexualidad para satisfacer la demanda corporal de sus parejas.

En la asociación con la variable edad del menor de los hijos, se esperaba encontrar que las mujeres que tienen hijos pequeños presentaran en mayor proporción deseo sexual inhibido, ya que es en esta edad cuando los hijos demandan mayor atención y por tanto mayor desgaste y cansancio de la madre. Sin embargo, esta disfunción se presenta con mayor frecuencia en mujeres que tienen hijos mayores de 13 años, lo que podría relacionarse con pérdida de intimidad, miedo a ser interrumpidos durante las relaciones y / o vergüenza a reconocer su sexualidad frente a sus hijos adolescentes. Al respecto, Laumann (1999) en su estudio concluyó que aquellas parejas con hijos que alteraban de alguna forma la intimidad de la pareja, reportaron índices más altos de disfuncionalidad sexual; se observa que existe una proporción mayor de deseo sexual inhibido en aquéllas que deben ocupar el dormitorio con otros miembros de la familia, lo que permite concluir que, sin ser estadísticamente significativa la asociación de estas variables, sí existe la influencia directa de la falta de intimidad en la aparición de disminución del deseo sexual. Referente al mismo punto, Mcabe (1997) concluye, luego de su investigación sobre intimidad, calidad de vida y disfuncionalidad sexual, que existe una asociación directa entre la intimidad y presencia de una disfunción sexual, ya que se encontró que aquellas mujeres disfuncionales sexualmente presentaron a su vez menor grado de intimidad.

En relación al uso de determinados anticonceptivos, se observa que no hay una diferencia significativa entre el dispositivo in- 
trauterino y los anticonceptivos orales. En aquellas mujeres que utilizaban preservativo en sus relaciones se reportó deseo sexual inhibido en un $44,4 \%$. Los resultados de este estudio difieren de aquellos obtenidos por Díaz y col. (1997), quienes encontraron que el uso de determinado método anticonceptivo puede influir positiva o negativamente sobre la experiencia de las personas con respecto a su propia sexualidad o a la de su pareja, evidenciaron que un $50 \%$ de las mujeres que tenía problemas sexuales lo atribuían al uso de anticonceptivos, reportándose una percepción de mejoría de su vida sexual en aquellas que utilizaban dispositivo intrauterino $(30 \%)$.

En relación a la escolaridad de la mujer y la de su pareja, los resultados muestran que existe una asociación estadísticamente significativa entre las variables en estudio, el deseo sexual inhibido se presenta en menor porcentaje en mujeres de mayor escolaridad. Se esperaba que hubiese una diferencia mayor entre aquellas mujeres que sólo tenían escolaridad básica con aquellas que tenían escolaridad media completa o incompleta; los resultados coinciden con el estudio dirigido por Laumann y col. (1999), quienes concluyeron que aquellas mujeres que no terminaron sus estudios presentaban en un $42 \%$ deseo sexual disminuido, y aquellas que sí los terminaron reportaron en un $24 \%$ alteración en el deseo sexual.

Respecto a la ocupación de la mujer, la literatura menciona que una actividad rutinaria en el hogar aumentaría la posibilidad de un desajuste en la parte sexual, lo que no se puede corroborar con el estudio presente, ya que no hay una diferencia importante en los porcentajes de deseo sexual inhibido presentado por ambos grupos de mujeres, a diferencia de la situación laboral de la pareja; alrededor del $50 \%$ de las mujeres cuyas parejas están cesantes presentan deseo sexual inhibido, lo que coincide con lo encontrado por Laumann (1999), quien concluye que menores ingresos económicos inciden en una mayor frecuencia de deseo sexual bajo. Sumado a lo anterior, aquellas mujeres que perciben como insatisfechas sus necesidades vitales básicas, presentan en mayor porcentaje deseo sexual inhibido $(44,6 \%)$, la asociación con la variable deseo sexual inhibido es estadísticamente significativa. En relación a la fuente de información sobre sexualidad, no existe significancia estadística para la asociación de la variable dependiente y las distintas fuentes de información. Se destacan, con un mayor porcentaje, los compañeros de estudios, los profesionales del área de la salud y los medios de comunicación. Estos resultados difieren de aquellos obtenidos en el "Estudio Nacional de Comportamiento Sexual", donde los profesionales del área de la salud como interlocutores en temas de sexualidad aparecen en un porcentaje bajo $(1,8 \%)$, la pareja en un $42 \%$ y amigos $26 \%$. Una educación inadecuada o inexistente contribuye a desarrollar mitos en relación a la sexualidad, vuelve más vulnerable a la persona a padecer una disfunción sexual y favorece además la persistencia de ella, tanto en el sexo femenino como masculino.

Con respecto a la variable religión, la diferencia en los porcentajes es destacable, sin embargo la asociación entre ambas variables no es estadísticamente significativa. La religión ha estado ligada históricamente a la formación de mitos y creencias no siempre verdaderas, en especial cuando se refiere a los comportamientos relacionados con la sexualidad, tales como masturbación, iniciativa, fantasías sexuales, etc. Del mismo modo, las construcciones culturales que existen sobre la sexualidad y su expresión han sido influenciadas por las diversas religiones, lo que ha contribuido a la sumisión y represión de los cuerpos, especialmente de las mujeres, no permitiendo vivir el placer en forma espontánea y natural como una dimensión básica del ser humano (Martín-Cano, F., 2002).

En relación a la percepción que tienen las mujeres de la satisfacción con su vida sexual independiente de la relación de pareja, llama la atención aquellas mujeres que, a pesar de presentar alteración del deseo sexual, tienen una vida sexual satisfactoria; esto puede estar relacionado con la conformidad de 
la mujer frente a su vida sexual, como lo evidenció Andrea Rodó (1987) en su estudio con mujeres pobladoras en Santiago, concluyendo que sólo un tercio de las mujeres estudiadas estaba satisfecha sexualmente. En relación a la satisfacción de la mujer con su relación de pareja independiente de la vida sexual, este estudio refleja que es estadísticamente significativa la asociación con la percepción del deseo sexual inhibido. Al respecto Fortin (1994) observó que la satisfacción marital autopercibida por la mujer estaba directamente relacionada con: su satisfacción sexual, la frecuencia de las relaciones sexuales, la satisfacción sexual percibida por la pareja y especialmente por la percepción de intimidad. En forma similar, Sanchez y col. (1997) concluyeron que las disfunciones sexuales se encuentran estrechamente relacionadas con un bajo autoconcepto, así como la disminución en la satisfacción marital.

Deseo sexual inhibido según satisfacción sexual

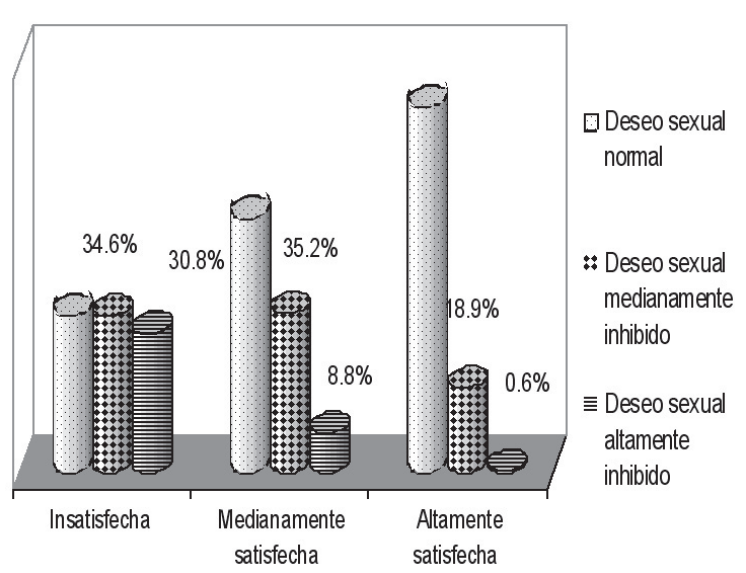

\section{CONCLUSIONES}

Alrededor de un tercio de las mujeres en edad fértil y que se controlan en los policlínicos de planificación familiar, presentan deseo sexual inhibido: $28,1 \%$ medianamente inhibido y un 6,8\% altamente inhibido.

-Las variables: edad de la mujer encuestada, de la pareja, del menor de sus hijos, espacio para la intimidad en la relación de pareja, uso de determinado método anticonceptivo, ocupación de la mujer, duración de la relación y religión que profesa, no se relacionan en forma estadísticamente significativa con la variable en estudio, deseo sexual inhibido.

-Existe relación estadística significativa entre la variable deseo sexual inhibido y las variables: escolaridad de la mujer, escolaridad de la pareja, situación laboral de la pareja, satisfacción de las necesidades básicas, satisfacción sexual y satisfacción con la relación de pareja.

Deseo sexual inhibido según satisfacción en la relación de pareja

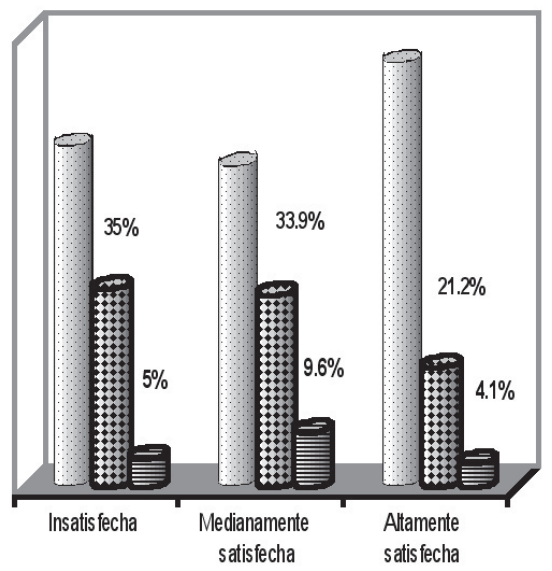

Deseo sexual normal

a: Deseo sexual medianamente inhibido

豆 Deseo sexual altamente inhibido

Fuente: Prevalencia de Deseo Sexual Inhibido y factores relacionados. Mónica Charnay, 2003.

\section{RECOMENDACIONES}

En el área asistencial: Los resultados de este estudio nos muestran la realidad local, de la prevalencia del deseo sexual inhibido, lo cual permitirá realizar una intervención clara y precisa en aquellas mujeres que debemos atender en los distintos lugares del quehacer de nuestra profesión.

En el área educativa: En la enseñanza de pregrado, la aplicación del instrumento uti- 
lizado en esta tesis facilitará la recolección de la información relacionada con la respuesta sexual y los factores relacionados con una probable disfunción.

En el área investigativa: Considerando los resultados obtenidos en este estudio, se debe crear nuevos conocimientos científicos sobre disfuncionalidad sexual, salud sexual, sexualidad de la mujer y de su pareja.

\section{REFERENCIAS BIBLIOGRAFICAS}

ABRANTES, M.; FREITAS, M.; DIMENSTEIN, M. (2001). Discurso sobre sexualidad y salud reproductiva en usuarias del Programa de Atención Integral a la Salud de la Mmujer en el Municipio de Natal, Brasil. Revista Mujer Salud. Red de Salud de las mujeres Latinoamericanas y del Caribe. Vol 2, pp. 3-15.

ALIAGA, P.; AHUMADA, S.; VILLAGRÁN, O.; SANTAMARÍA, M.;MANZOR, S.; ROJAS, O. (2000). Disfunciones sexuales: asistencia clínica y factores asociados en ginecología. Revista Chilena Obstetricia y Ginecología Vol. LXV N 6, pp. 442-452.

BARRA, E. (1999). Psicología de la sexualidad. Proyecto de Docencia N ${ }^{\circ}$ 97-156. Ed. Facultad de Ciencias Biológicas. Universidad de Concepción. Chile.

CARROBLES, J. A. (1990). Biología y psicofisiología de la conducta sexual. Fundación Universidad-Empresa. Madrid.

DÍAZ, M.; MOORE, K. (1999). La evolución de un programa educativo sobre sexualidad. En S. Zeidenstein y K. Moore (editoras), Aprendiendo sobre sexualidad. Una manera práctica de comenzar (pp. 225240). Nueva York.

DIXON-MUELLER, RUTH (1999). Las conexiones entre sexualidad y salud reproductiva. En S. Zeidenstein y K. Moore (editoras), Aprendiendo sobre sexualidad. Una manera práctica de comenzar (pp. 157-180). Nueva York

FLORES, A. (2000). Disfunciones y terapias sexuales del 2000. ( $5^{\circ}$ edición). A\&M ediciones Montevideo, Uruguay.

FORTIN, NATHALIE \& THÉRIAULT, J. (1994). Intimité el satisfaction sexualle. Revue sexologique vol. 3 $\mathrm{N}^{\circ}$ 1. Obtenido en: http://www.unites.uqam.ca/ dsexo/Revue/Vol3no1

HAWTON, K. (1988). Terapia sexual. Ed. Doyma. España.

JIMÉNEZ, E. (1999). Aprendiendo juntas. En S. Zeidenstein y K. Moore (editoras), Aprendiendo sobre sexualidad. Una manera práctica de comenzar (pp. 38-50). Nueva York.

LABRADOR, F. (1994). Guía de la sexualidad. Espasa Calpe. España.

LAUMANN, E \& PAIK, A; ROSEN, C. (1999). Sexual dysfuntion in the United States: prevalence and predictors. The University of Chicago Chronicle, vol $18, \mathrm{~N}^{\circ} 10$.

MARTINEZ, R. (1999). Psicosexualidad y conducta humana. Proyecto de desarrollo de docencia 97-155, Ed. Facultad de Ciencias Biológicas. Universidad de Concepción. Chile.

MARTÍN-CANO ABREU, FRANCISCA (2002). Comparación entre sexualidad humana y animal. Revista Familia. Revista Cutural y Educativa, $\mathrm{N}^{\circ} 13$ : sept. 15 - octu. 15. Toronto, Ontario, Canadá http:/ www.revistafamilia.com/issue $013 / 13$ comportamiento humano.htm

MASLOW, A J. (1963). Motivación y personalidad. Ed. Sagitario. Barcelona.

MASLOW, A J. (1985). El hombre autorrealizado. (6 ${ }^{\circ}$ Ed.). Kairos. Buenos Aires. Argentina.

MASTER, W.; JOHNSON, V; KOLODNY, R. (1996). Eros los mundos de la sexualidad (13 ed.) Grijalbo. Barcelona.

MCABE, MP. (1997). Intimacy and quality of life among sexually dysfunctional men and women Journal Sex Marital Ther, vol 23, $n^{\circ} 4$, pp. 276-90.

MINISTERIO DE SALUD (2002). Comisión Nacional del Sida Estudio Nacional de Comportamiento Sexual. Chile.

MORRIS, D. (2000). Masculino y femenino ( $1^{\circ}$ ed.). Plaza \& Janés ed. Barcelona.

PINO, E; URQUIETA, M. (1992). El Arte del equilibrio erótico. Ed. Planeta. Chile.

OMS. Promoción de la salud sexual. Recomendaciones para la acción (2000). Actas reunión de consulta por OPS y OMS. Guatemala. Obtenido en http:/ www2.rz-hu-berlin.de / sexology / GESUND / ARCHIV / FIRST.HTM

PALACIOS, P.; MONTECINO, S.; REBOLLEDO, L. (1995). Mujer y género. Nuevos saberes en las universidades chilenas. Bravo y Allende editores. Colección de Ciencias Sociales. Universidad de Chile. Chile.

RIVERA, FIGUEROA (1993). Cuadernos de Capacitación en Investigación sobre Planificación Familiar. Santiago. Chile.

RODÓ, ANDREA (1987). El cuerpo ausente, Revista Proposiciones $\mathrm{N}^{\mathrm{o}} 13$ enero-abril. Chile.

ROSENZVAIG, ROBERTO (1994). La pareja al desnudo. Ed. Sudamericana, Buenos Aires. Argentina.

SARQUIS, C. (1993). Introducción al estudio de la pareja humana. Ed. Universitaria S.A. U. Católica de Chile. Chile.

SÁNCHEZ-BRAVO C. y CARREÑO-MELÉNDEZ, J.; GONZÁLEZ-CAMPILLO, G.; GONZÁLEZ-VALENCIA, I. (1997). Autoconcepto y satisfacción marital en mujeres con disfunción sexual. Perinatol Reprod Hum; 11(4): 190-197.

WORTH, DOOLEY (1999). ¿Qué tiene que ver el amor en esto? La influencia del amor romántico en la conducta sexual de riesgo. En S. Zeidenstein y K. Moore (editoras), Aprendiendo sobre sexualidad. Una manera práctica de comenzar (pp. 135-155). Nueva York.

ZEIDENSTEIN, S. Y MOORE, K. (1999). Aprendiendo sobre sexualidad. The Population Council. Nueva York. 